\title{
Defective concept formation in Parkinsonians is independent from mental deterioration
}

\author{
CARLO CALTAGIRONE, AUGUSTO CARLESIMO, UGO NOCENTINI, \\ STEFANO VICARI \\ From the Clinica Neurologica, II Universitá di Roma, Rome, Italy
}

SUMMARY An extensive neuropsychological battery (the Mental Deterioration Battery) was utilized to distinguish, within a sample of 24 idiopathic Parkinsonians, those showing signs of diffuse mental deterioration $(n=9)$ from those without deterioration $(n=15)$. Performances of control subjects on a wide range of tests exploring mnesic, visuo-constructive, linguistic and general intellectual functions $(n=21)$ did not differ from analogous performances of Parkinsonians without signs of diffuse mental deterioration. The Wisconsin Card Sorting Test was then used to verify the hypothesis that a selective impairment of cognitive functions subsumed by the integrity of frontal lobes could be demonstrated in Parkinsonian patients. Our results provide evidence that in this task, defective performances are obtained by Parkinsonians and even by patients without signs of diffuse cognitive impairment. These findings seem to confirm that a deficit in concept formation, maintenance and shifting is largely independent of the dementia frequently noticed in Parkinson's disease.

It is no longer disputed that there is a prevalence of dementia in patients affected by Parkinson's disease which is higher than in the normal population. ${ }^{1}$ Moreover, even when diffuse mental deterioration has been absent in Parkinsonian patients, selective deficits of cognition may be found. ${ }^{2}$ Some authors claim that an asymmetrical pattern of cognitive performances can be found in Parkinsonians with worst scores on visuo-spatial tasks. ${ }^{34}$ However, this selective impairment of visuo-spatial functions has not been confirmed when the motor-speed component of these tasks is taken into account. ${ }^{25}$

Impairment of mnesic functions seems to be better documented. Long term recall of visual and verbal material seems to be affected even in the presence of normal scores on tasks of short term memory and long term storage. ${ }^{67}$

After the description by Bowen et al of a selective disorder in concept formation in Parkinsonians, many authors have described these patients as particularly compromised in tests sensitive to lesions of frontal lobes. ${ }^{9-11}$

In any case, before positing that a selective impairment of cognitive functions is actually present in

Address for reprint requests: Prof $\mathrm{C}$ Caltagirone, Institute of Neurology, II Universitá di Roma, Osp S Eugenio, P le dell'Umanesimo 10, 00144 Roma, Italy.

Received 19 January 1988 and in final revised form 8 August 1988. Accepted 26 August 1988
Parkinsonians, the weight of a more general mental deterioration should be checked. In most of the studies pointing to an impairment of frontal functions in patients affected by Parkinson's disease this factor has not convincingly been ruled out. The presence of a more global cognitive impairment has generally been excluded on the basis of the IQ derived from the WAIS, while a detailed survey of mnesic, visuoconstructive and general intellectual functioning is often missing. ${ }^{810}$

To verify the hypothesis that there is a selective deficit of functions normally subsumed by the integrity of frontal lobes in Parkinsonians, we planned a study in which a careful evaluation of mental functioning was performed together with a specific assessment of concept formation, maintenance and shifting.

\section{Material and methods}

\section{Neuropsychological assessment}

To assess the presence of a general impairment of mental functions we used the Mental Deterioration Battery (MDB). This consists of eight tasks capable of yielding distinctive information about mnesic, linguistic, visuo-constructive and general intellectual functions. The battery has been extensively described in a previous study. ${ }^{12}$ In table 1 the single MDB tests and the corresponding cognitive functions investigated are summarised. As demonstrated in a previous paper, ${ }^{12}$ the MDB is able to classify correctly normal and deteriorated subjects on the basis of the number of pathological performances. A patient is classified as demented 
Table 1 Tasks used to build up the MDB: the corresponding cognitive functions investigated by the single tests are indicated

\begin{tabular}{ll}
\hline $\begin{array}{l}\text { Word Fluency (WF) } \\
\text { Phrase Construction (PC) }\end{array}$ & Verbal attainments \\
$\begin{array}{l}\text { Rey's Verbal short term recall (RST) } \\
\text { memory test }\end{array}$ & Verbal memory term recall (RLT) \\
$\begin{array}{l}\text { Immediate Visual Memory (IVM) } \\
\begin{array}{l}\text { Coloured Raven's } \\
\text { Progressive Matrices (PM'47) }\end{array}\end{array}$ & $\begin{array}{l}\text { Visuospatial Memory } \\
\text { on visuospatial data }\end{array}$ \\
$\begin{array}{ll}\text { Copying designs free copy (CD) } \\
\text { copy with landmarks } \\
\text { (CDL) }\end{array}$ & $\begin{array}{l}\text { Visuoconstructive } \\
\text { functions }\end{array}$ \\
\hline
\end{tabular}

when he obtains four or more pathological performances on the eight tasks of the MDB. The cutting scores differentiating normal from pathological performances are derived from previously published normative data. ${ }^{12}$

The test used to assess the ability of concept formation, maintenance and shifting was the Wisconsin Card Sorting Test (WCST), which was administered in full measure (128 cards) according to the procedure employed by Milner. ${ }^{13}$ In the evaluation of the results we considered the categories obtained together with the number of perseverative and total errors produced.

As originally described by Milner, ${ }^{13}$ and subsequently confirmed by other authors ${ }^{1415}$ patients with frontal lobe damage do particularly badly in this test. In these studies the performance of patients with frontal lobe damage was indicative of a defective acquirement of categories, and exhibited a perseverative behaviour in comparison with both normal subjects and patients with lesions restricted to posterior regions of the brain.

\section{Subjects}

Twenty four consecutive outpatients of the Neurological Clinic of our University showing clinical evidence of idiopathic Parkinson's disease were included in our study. None of them had clinical signs of cerebrovascular disease, alcoholism or depression nor had they undergone thalamotomy or other surgical procedures for the relief of Parkinsonian symptoms.

The mean length of the illness was $4 \cdot 1$ years (SD 1-4). All the patients demonstrated a level of disability between stages II and III of the Hoehn and Yahr scale. ${ }^{16}$

Eighteen Parkinsonian patients were in a pharmacological wash-out period; the others were receiving therapy with levodopa alone or associated with anticholinergics.

Twenty one subjects, most of them relatives of the Parkinsonian subjects, without clinical evidence of

Table 2 Age and literacy levels of control and Parkinsonian subjects

\begin{tabular}{lccll}
\hline & $\begin{array}{l}\text { Controls } \\
(n=21)\end{array}$ & $\begin{array}{l}\text { Parkinsonians } \\
(n=24)\end{array}$ & $t$ & $p$ \\
\hline $\begin{array}{l}\text { Age (years) } \\
\begin{array}{l}\text { Years of } \\
\text { schooling }\end{array}\end{array}$ & $59.6(7.8)$ & $61.5(7.6)$ & 0.81 & NS \\
\hline
\end{tabular}

Table 3 Mean scores (and standard deviations) normalised in z points obtained on various MDB tasks by control subjects and Parkinsonian patients; statistical comparisons on Student's $t$ test are also reported

\begin{tabular}{lllll}
\hline & $\begin{array}{l}\text { Controls } \\
(n=21)\end{array}$ & $\begin{array}{l}\text { Parkinsonians } \\
(n=24)\end{array}$ & $t$ & $p$ \\
\hline WF & $0.16(1.0)$ & $-0.14(0.95)$ & 1.0 & NS \\
CF & $0.15(1.0)$ & $-0.13(0.97)$ & 0.99 & NS \\
RST & $0.31(1.0)$ & $-0.27(0.89)$ & 2.05 & $<0.05$ \\
RLT & $0.17(0.97)$ & $-0.14(1.0)$ & 1.08 & NS \\
IVM & $0.23(0.84)$ & $-0.19(1.0)$ & 1.04 & NS \\
PM'47 & $0.15(0.86)$ & $-0.13(1.1)$ & 0.96 & NS \\
CD & $0.25(0.79)$ & $-0.21(1.1)$ & 1.62 & NS \\
CDL & $0.23(0.58)$ & $-0.20(1.2)$ & 1.49 & NS \\
\hline
\end{tabular}

neurologic or psychiatric disorders were used as the control group.

\section{Results}

Although the age and literacy levels demonstrated by Parkinsonians and control subjects were comparable (table 2), performance scores for the two groups on the various tasks of the MDB were adjusted for age and years of schooling on the basis of the standard procedures reported in a previous paper. ${ }^{12}$ Moreover, so that results obtained in tests having different ranges could be compared, the adjusted scores were transformed into normalised $\mathrm{Z}$ scores.

The results obtained on various MDB tests by both Parkinsonian and control groups are summarised in table 3.

Parkinsonian patients obtained lower scores than control subjects on most of the tasks. A two-way analysis of variance, in which the results obtained in the eight MDB tests were taken into consideration, reveals that the difference between the two groups approaches the level of significance $(F=3.6 ; p=$ $0.06)$. These results are in agreement with the data of several other authors. ${ }^{61718}$

Brown and Marsden ${ }^{2}$ suggested that in non-selected

Table 4 Mean scores (and standard deviations) normalised in $z$ points obtained on various $M D B$ tasks by control subjects and non-demented Parkinsonian patients; statistical comparisons on Student's t test are also reported

\begin{tabular}{lllll}
\hline & $\begin{array}{l}\text { Controls } \\
(n=21)\end{array}$ & $\begin{array}{l}\text { Non-demented } \\
\text { Parkinsonians } \\
(n=15)\end{array}$ & $t$ & $p$ \\
\hline WF & $0.16(1.0)$ & $0.10(0.91)$ & 0.20 & NS \\
CF & $0.15(1.0)$ & $0.36(0.74)$ & 0.64 & NS \\
RST & $0.31(1.0)$ & $0.18(0.75)$ & 0.41 & NS \\
RLT & $0.17(0.97)$ & $0.29(0.83)$ & 0.39 & NS \\
IVM & $0.23(0.84)$ & $0.33(0.69)$ & 0.40 & NS \\
PM'47 & $0.15(0.86)$ & $0.40(0.95)$ & 0.80 & NS \\
CD & $0.25(0.79)$ & $0.27(0.80)$ & 0.05 & NS \\
CDL & $0.23(0.58)$ & $0.35(0.82)$ & 0.61 & NS \\
\hline
\end{tabular}




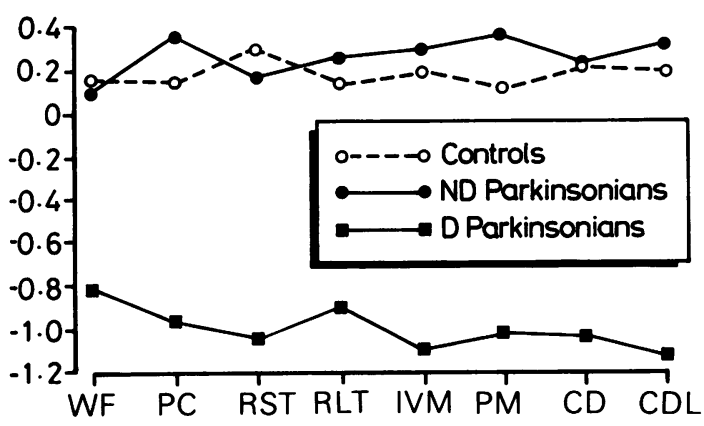

Fig Neuropsychological profiles exhibited on the MDB by our samples of control subjects and Parkinsonian patients affected (D) or not (ND) by mental deterioration.

populations mean performance values may be affected significantly by the inclusion of subjects suffering from mental deterioration. Since the purpose of our study was to detect a possible selective deficit in cognition in Parkinsonian patients without clinical and neuropsychological signs of dementia, we subdivided the total sample of Parkinsonians into two groups. On the basis of MDB performance, the patients of the total sample were identified as being affected or not by mental deterioration. The first group was composed of 15 patients classified as not deteriorated while the remaining nine patients, classified as deteriorated, made up the second group. The prevalence of dementia in our group of Parkinsonians is comparable with that reported in larger samples. ${ }^{19} 20$

The two subgroups of Parkinsonians did not differ as regards mean age (non deteriorated: $60 \cdot 0$, SD $8 \cdot 1$; deteriorated: $63 \cdot 5, \mathrm{SD} 6 \cdot 9 ; t=1 \cdot 1, \mathrm{p}=\mathrm{NS}$ ) and years of schooling (non deteriorated: 6.9, SD 2.2; deteriorated: $5 \cdot 7$, SD 2.0; $t=1 \cdot 4, \mathrm{p}=\mathrm{NS}$ ). Non deteriorated Parkinsonians had a mean duration of disease (3.6 years; SD 1.2) significantly shorter than deteriorated ones $(4 \cdot 8$ years; SD $1 \cdot 3)(t=2 \cdot 3 ; p=$ 0.03). Thirteen of the non deteriorated Parkinsonians were in a pharmacological wash-out period while the remaining two patients were receiving levodopa.

In the deteriorated group, five patients were in a pharmacological wash-out period. The remaining four patients were receiving levodopa alone (two patients) or in association with anticholinergics (two patients). All the control subjects demonstrated on the MDB a number of pathological performances below four and then were classified as non deteriorated.

A comparison of performances on the various MDB tasks obtained by control subjects and nondeteriorated Parkinsonians is reported in table 4. A two-way analysis of variance does not reveal any significant difference for any factor $(F$ always $<1)$. No significant difference can be detected even in the comparisons of single tests.

Profiles exhibited on the various tasks of MDB by control subjects and Parkinsonian patients are shown in the figure. Profiles of performances obtained by controls and non deteriorated Parkinsonians are almost superimposable while the profile shown by deteriorated Parkinsonians is quite different from these.

The WCST results for the three groups, control subjects, non-demented and demented Parkinsonians, are summarised in table 5 .

Given that performance scores obtained did not fit a normal distribution, statistical analysis was performed by means of a non parametric test (Mann-Whitney U test).

Non-deteriorated Parkinsonians produced significantly more perseverative errors and obtained fewer categories (this difference only approaches statistical significance: $p=0.07$ ) than control subjects. In the comparison between performance scores demonstrated by the two subgroups of Parkinsonians the difference regarding the number of categories and of perseverative errors approaches significance (categories: $p=0.05$; perseverative errors: $p=0.06$ ), while the difference in the number of total errors reaches the level of statistical significance. No significant difference was detected in the number of total errors between normal subjects and non-deteriorated Parkinsonians.

\section{Discussion}

Several authors have described impaired performances on "frontal tasks" in patients affected by Parkinson's disease which did not show clinical and neuropsychological signs of general intellectual deterioration. Bowen et $a^{\beta}$ found that Parkinsonian patients formed significantly fewer concepts, as measured by WCST, than controls matched for age,

Table 5 Medians (and ranges) of scores obtained on WCST by control subjects, non-deteriorated and deteriorated Parkinsonians; statistical comparisons on Mann-Whitney $U$ test are also indicated

\begin{tabular}{|c|c|c|c|c|c|c|c|}
\hline & $\begin{array}{l}\text { Control } \\
\text { subjects } \\
(n=21)\end{array}$ & $z$ & $p$ & $\begin{array}{l}\text { Non-demented } \\
\text { Parkinsonians } \\
(n=15)\end{array}$ & $z$ & $p$ & $\begin{array}{l}\text { Demented } \\
\text { Parkinsonians } \\
(n=9)\end{array}$ \\
\hline $\begin{array}{l}\text { Categories } \\
\text { Total errors } \\
\text { Perseverative errors }\end{array}$ & $\begin{array}{c}5 \cdot 8(3-6) \\
36 \cdot 0(1-72) \\
10 \cdot 0(0-52)\end{array}$ & $\begin{array}{l}1.8 \\
1.4 \\
2.0\end{array}$ & $\begin{array}{l}\text { NS } \\
\text { NS } \\
=0.04\end{array}$ & $\begin{array}{l}5 \cdot 0(1-6) \\
42 \cdot 0(14-82) \\
25 \cdot 0(3-71)\end{array}$ & $\begin{array}{l}1.9 \\
2.0 \\
1.8\end{array}$ & $\begin{array}{l}\text { NS } \\
=0.04 \\
\text { NS }\end{array}$ & $\begin{array}{c}2 \cdot 0(0-5) \\
58 \cdot 0(38-74) \\
34.0(22-40)\end{array}$ \\
\hline
\end{tabular}


literacy and verbal WAIS IQ. For Parkinsonian patients with normal IQ Lees and Smith' reported difficulty in shifting conceptual sets and a perseverative behaviour on both the modified WCST and Benton's Word Fluency test. A similar shifting aptitude disorder was observed in tests of block and animal sorting and finger pushing sequences. ${ }^{10}$

In these studies a general intellectual impairment was excluded on the basis of a normal WAIS IQ. Nevertheless, the WAIS, a battery of tests widely used for determining the intellectual level of normal subjects, appears to be rather unreliable for detecting initial forms of mental deterioration and unable to furnish a detailed survey of mnesic, visuoconstructive and linguistic functions. For this reason, the deficits observed in "frontal tasks" were conceivably part of an initial form of diffuse mental deterioration.

A more comprehensive survey of intellectual functions in clinically non-demented Parkinsonian patients has been reported by Taylor et al. ${ }^{11}$ In this study the patients were evaluated by means of tests of verbal and visual memory, visuo-spatial ability and executive functions. The Parkinsonian patients exhibited impaired performances on tasks of long term verbal recall, immediate logical memory and on a cluster of tests, such as WCST, Trail Making Test, Design Fluency and Delayed Recognition, thought to be sensitive to frontal lobe function.

The present research was designed to evaluate the hypothesis of a selective deficit of frontal functions in Parkinsonian patients in whom a more diffuse intellectual deterioration had previously been excluded. A detailed analysis of the MDB results shows that Parkinsonian patients classified as non-demented do not differ from normal subjects on tasks exploring mnesic, visuoconstructive, linguistic and general intellectual functions. Only on WCST did these patients exhibit pathological performances. In particular, they produced significantly more perseverative errors than normal controls and the difference between the number of categories obtained by these patients and by normal controls approaches significance although they appear less compromised than demented Parkinsonians in this task. For nondeteriorated Parkinsonian patients these results reveal diminished capacity for concept formation and maintenance and a disorder in cognitive shifting aptitude.

This neuropsychological pattern, characterised by an isolated deficit in a task thought to be sensitive to lesions of frontal lobes, seems to indicate a selective impairment of functions subsumed by frontal lobes in Parkinsonian patients even when a more diffuse involvement of cognitive functions has been ruled out.

These findings can be interpreted on the basis of histopathological data suggesting, in Parkinson's disease, an alteration in afferent pathways from basal ganglia and midbrain gray nuclei to frontal cortex. The "complex loop" connecting frontal lobes to basal ganglia can be disrupted by a reduction of dopaminergic activity in the caudate nucleus of Parkinsonian patients. " Furthermore, a reduction of dopamine content and of its metabolites in frontal cortical areas has been found in the brain of Parkinsonian subjects. ${ }^{21}$ This finding is probably related to the degeneration of a mesocortical dopaminergic pathway projecting from the mesencephalon to cingulate, entorhinal and frontal cortex..$^{22}$

\section{References}

1 Brown RG, Marsden CD. How common is dementia in Parkinson's disease? Lancet 1984;ii:1262-5.

2 Brown RG, Marsden CD. Neuropsychology and cognitive functions in Parkinson's disease: an overview. In: Marsden CD, Fahn S eds. Movement Disorders 2. London: Butterworth 1986:99-123.

3 Meier MJ, Martin WE. Intellectual changes associated with levodopa therapy. JAMA 1970;213:465-6.

4 Loranger AW, Goodel H, McDowell FH, Lee JE, Sweet RD. Intellectual impairment in Parkinson's syndrome. Brain 1972;95:405-12.

5 Brown RG, Marsden CD. Visuospatial function in Parkinson's disease. Brain 1986;109:987-1002.

6 Pirozzolo FJ, Hansch EC, Mortimer JA, Webster DD, Kuskowski MA. Dementia in Parkinson's disease: a neuropsychological analysis. Brain Cogn 1982;1:71-83.

7 Weingartner H, Burns S, Diebel R, Le Witt PA. Cognitive impairment in Parkinson's disease: distinguishing between effort-demanding and automatic cognitive processes. Psychiatry Res 1984;ii:223-35.

8 Bowen FP, Kamienny RS, Burns MM, Yahr MD. Parkinsonism: effects of levodopa on concept formation. Neurology 1975;25:701-4.

9 Lees AR, Smith E. Cognitive deficits in early stages of Parkinson's disease. Brain 1983;106:257-70.

10 Cools AR, Van Den Bercken JHL, Horstink MWI, Van Spaendonck KPM, Berger HJC. Cognitive and motor shifting aptitude disorder in Parkinson's disease. J Neurol Neurosurg Psychiatry 1984;47:443-53.

11 Taylor AE, Saint-Cyr JA, Lang AE. Frontal lobe dysfunction in Parkinson's disease. The cortical focus of neostriatal outflow. Brain 1986;109:845-83.

12 Caltagirone C, Gainotti G, Masullo C, Miceli G. Validity of some neuropsychological tests in the assessment of mental deterioration. Acta Psychiatr Scand 1979;60:50-56.

13 Milner B. Effects of different brain lesions on card sorting: the role of the frontal lobes. Arch Neurol 1963;9:90-100.

14 Nelson HE. A modified card sorting test sensitive to frontal lobe effects. Cortex 1976;12:313-24.

15 Robinson AL, Heaton RK, Lehman RA, Stilson DW. The utility of the Wisconsin Card Sorting Test in detecting and localising frontal lobe lesions. J Consult Clin Psychol 1980;48:605-14.

15 Hohen NM, Yahr MD. Parkinsonism: onset, progression and mortality. Neurology 1967;17:427-42.

17 Reitan RM, Boll TJ. Intellectual and cognitive functions in Parkinson's disease. J Consulting Clin Psychol 1971;37:364-9.

18 Portin R, Rinne UK. Neuropsychological responses of Parkinsonian patients to long-term levodopa therapy. In: Klinger $\mathbf{M}$, Stamm G, eds. Parkinson's disease: Current Progress, Problems and Management. Amsterdam: Elsevier/North Holland 1980:271-304.

19 Boller F, Mizutani T, Roessmann U, Gambetti P. Parkinson's disease, dementia and Alzheimer disease: clinico-pathological correlations. Ann Neurol 1980;1:329-55.

20 Caltagizone C, Masullo C, Benedetti N, Gainotti G. Dementia in Parkinson's disease: possible specific involvement of the frontal lobes. Int J Neurosci 1985;26:15-26.

21 Scatton B, Rouquier L, Javoy-Agid F, Agid Y. Dopamine deficiency in the cerebral cortex in Parkinson disease. Neurology 1982;32:1039-40.

22 Javoy-Agid F, Agid Y. Is the mesocortical dopaminergic system involved in Parkinson disease? Neurology 1980;30:1326-30. 